\title{
On the response of ionospheric electrojets to solar wind discontinuities
}

\author{
M. Palmroth ${ }^{1}$, T. I. Pulkkinen ${ }^{1}$, J. Polvi ${ }^{2}$, A. Viljanen ${ }^{1}$, and P. Janhunen ${ }^{1}$ \\ ${ }^{1}$ Finnish Meteorological Institute, Helsinki, Finland \\ ${ }^{2}$ University of Helsinki, Helsinki, Finland
}

Received: 4 March 2009 - Revised: 7 August 2009 - Accepted: 22 September 2009 - Published: 6 October 2009

\begin{abstract}
We investigate the ionospheric response to solar wind discontinuities as detected by the $I E$ index computed from IMAGE ground magnetometers. The solar wind discontinuities include both sudden increases as well as decreases of the solar wind dynamic pressure, recorded by the SWEPAM instrument of the ACE spacecraft during the period 1998-2004. In our statistical study, we identify four categories of events: 1) sudden increases of the dynamic pressure with a simultaneous increase of the interplanetary magnetic field (IMF) magnitude; 2) sudden increases of the dynamic pressure accompanied with a simultaneous decrease of the IMF; 3) sudden decreases of the dynamic pressure accompanied with a sudden increase of the IMF; and 4) sudden decreases of the dynamic pressure with relatively steady IMF. We perform a superposed epoch analysis for the four event categories to distinguish the ionospheric response. We find that the $I E$ index increases/decreases in response to the solar wind dynamic pressure increases/decreases regardless of the simultaneous change in the IMF or the amount of estimated input energy. We investigate the magnitude of the ionospheric response according to the IMF north-south direction, the dynamic pressure step size as well as the pressure level prior the dynamic pressure change. We find that the ionospheric result is augmented for larger pressure steps, while the prior IMF has a role only in some of the event categories. We also perform global MHD simulation runs to investigate the ionospheric dissipation rate during such solar wind discontinuities, and find that the simulation results are in good qualitative accordance with the observational statistical results.
\end{abstract}

Keywords. Ionosphere (Electric fields and currents) Magnetospheric physics (Magnetosphere-ionosphere interactions; Solar wind-magnetosphere interactions)

Correspondence to: M. Palmroth (minna.palmroth@fmi.fi)

\section{Introduction}

The global energetics of the near-Earth space system is one of the most important open questions in space physics, and one that is markedly difficult to study using local insitu observations. It is widely accepted that the interplanetary magnetic field (IMF) plays a crucial role in solar wind-magnetosphere-ionosphere coupling (Dungey, 1961), and hence the IMF is always present in the various proxies to estimate the energy input. A well-known example is the $\epsilon=\left(4 \pi / \mu_{0}\right) v B^{2} l_{0}^{2} \sin ^{4}(\theta / 2)$ parameter (Akasofu, 1981), where $\mu_{0}$ is the vacuum permeability, $v$ is the solar wind speed, $B$ is the IMF magnitude, $l_{0}$ is a scaling length, and $\theta$ is the IMF clock angle in the $Y Z_{\mathrm{GSM}}$ plane. The solar wind dynamic pressure, given as $P=\rho v^{2}$, where $\rho$ is the solar wind mass density, is present in $\epsilon$ only through the solar wind velocity.

In addition to the IMF, the solar wind dynamic pressure is another important driver of magnetospheric dynamics, whose effects have been studied to lesser extent over the years. The immediate reaction to a sudden increase in the solar wind pressure is the intensification of the ChapmanFerraro currents at the magnetopause and the compression of the magnetosphere (Baumjohann et al., 1983). The compression induces a magnetic perturbation at the dayside low latitudes (Araki, 1976), which have been termed as sudden impulses (SI) or storm sudden commencements (SSC), which are dynamic pressure enhancements preceding some (but not all) magnetic storms. A sudden decrease of the solar wind dynamic pressure leads to expansion of the magnetosphere and is also observed in the equatorial ground magnetic field (Araki and Nagano, 1988).

In the past few years, the solar wind dynamic pressure has gained more interest as a driver of magnetospheric dynamics: The dynamic pressure variations can modulate reconnection at the magnetopause (Lu et al., 2004) and in the magnetospheric tail (Hubert et al., 2006). Enhanced tail reconnection as a response to a dynamic pressure pulse is observed as a

Published by Copernicus Publications on behalf of the European Geosciences Union. 
decrease of the polar cap area (Zesta et al., 2000; Boudouridis et al., 2005). Shue and Kamide (2001) were the first to identify that the large solar wind density enhances the auroral electrojets during magnetic storms. Lopez et al. (2004) interpreted this density effect by the larger compression ratio in the magnetosheath during intense southward IMF, indicating that the actual magnetic field in contact with the terrestrial field is more intense during times of high solar wind densities.

Liou et al. (2004) investigated the intensifications of the westward electrojets in the ionosphere associated with $43 \mathrm{dy}-$ namic pressure enhancements. Their primary target was to quantify the preconditioning hypothesis stating that the solar wind dynamic pressure enhancements can trigger substorms and release previously stored energy if the magnetosphere has been "preconditioned" by southward IMF prior the shock arrival. Liou et al. (2004) found that the intensity of the westward electrojets as measured by the $A L$ index is much better correlated with the postshock rather than preshock solar wind parameters, bringing doubt over the preconditioning hypothesis. Essentially, Liou et al. (2004) concluded that after dynamic pressure enhancements the magnetosphere is directly driven, and that the solar wind dynamic pressure plays at least an equally important role as the southward IMF when the geoefficiency of solar wind parameters is considered.

While the sudden increases of the dynamic pressure has gained more attention recently, the magnetospheric response to sudden decreases of the dynamic pressure has not yet been studied in detail. Lukianova (2003) and Stauning and Troshichev (2008) found that for negative pressure steps the temporal evolution of the polar cap index (PCI) is consistent with transient convection vortices that divert ChapmanFerraro currents from the magnetopause to the polar cap ionosphere. In a case study, Sato et al. (2001) found that the local auroral brightness as measured by an all-sky-camera can increase after a negative pressure impulse. Liou et al. (2006) investigated 13 sudden decreases of the dynamic pressure from Polar Ultraviolet Imager (UVI) and found that globally both the auroral luminosity as well as the average energy of precipitating electrons decrease as a response. They noted that the result is not in contradiction with Sato et al. (2001) as in some cases local brightenings did occur also in the global UVI pictures. Liou et al. (2006) speculated that the decrease of the global luminosity may be explained by an adiabatic expansion and reduction of the mirror ratio of the precipitating electrons, while possibly induction electric fields and Fermi acceleration effects would be the cause of the decreasing energy of the loss cone electrons.

An interesting yet unsolved question is whether the dynamic pressure changes can have a measurable effect in the global energy partitioning and total ionospheric energy dissipation. Shue et al. (2009) investigated the auroral electrojet and brightness response to a solar wind density step as a sole upstream driver. They concluded that while the electrojets enhance, the total auroral power may not significantly increase after the density step. In a global MHD simulation of a magnetic storm, Palmroth et al. (2003) noticed that the Joule dissipation rate in the simulation ionosphere resembles the time evolution of the solar wind dynamic pressure. Observationally, Palmroth et al. (2004) noted an increase of the $A E$ index as a response to dynamic pressure impulses in a small statistical study. They suggested that as the ChapmanFerraro currents at the magnetopause are connected with the Region 1 currents, the magnetopause current intensification resulting from a dynamic pressure increase would be observed as higher Joule dissipation rates in the ionosphere.

In this paper we investigate the ionospheric response to abrupt changes in the solar wind dynamic pressure. We identify both sudden increases and decreases of the solar wind dynamic pressure, and evaluate the response in the ionosphere statistically by the Joule dissipation rate. As the Joule dissipation rate originates from the ionospheric closure of the field-aligned currents, the magnitude of the horizontal currents is proportional to Joule dissipation. Hence, we estimate the dissipation using the $I E$ index computed from the IMAGE ground magnetometers. We also carry out global MHD simulation runs to investigate the ionospheric dissipation in the simulation ionosphere. The paper is organized as follows: First, we present our data set and event selection criteria. Then, we investigate the ionospheric response during different circumstances using superposed epoch statistics. Finally, we end the paper with our discussion and conclusions.

\section{Data set}

In this report we extend the data set of Palmroth et al. (2007), where the ionospheric response to solar wind dynamic pressure impulses was studied in a superposed epoch analysis. In addition, we establish a new data set to investigate the ionospheric response to sudden decreases of the dynamic pressure, termed negative pressure pulses or steps. The upstream observations are recorded by the ACE spacecraft: the solar wind density and velocity are determined by the Solar Wind Electron Proton Alpha Monitor (SWEPAM) instrument (McComas et al., 1998), while the IMF observations are recorded by the magnetic field instrument (MAG) (Smith et al., 1998). The ionospheric response is determined from the IMAGE magnetometer array ranging from Svalbard to Southern Estonia ( $\sim 55-75$ magnetic latitude). We compute a local $I E$ index, which is defined as the difference between the maximum and minimum of the northward magnetic field perturbation, in a manner similar in which the $A E$ index is calculated. The $I E$ index measures the total current in the east-west direction above the IMAGE chain with good accuracy (Vanhamäki et al., 2003), with a thumb rule of about $1 \mathrm{nT}$ corresponding to $1000 \mathrm{~A}$. 

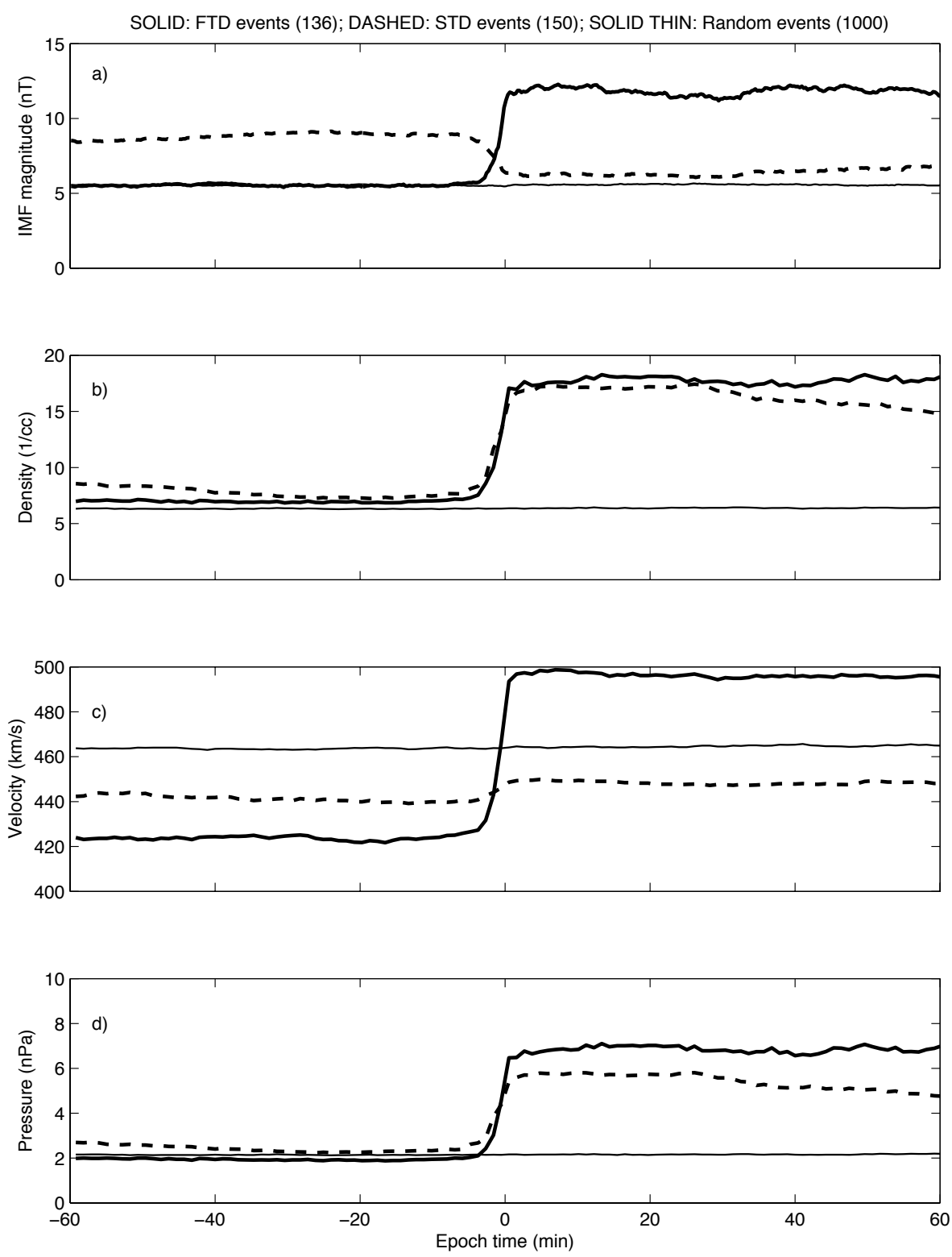

Fig. 1. Superposed epoch averages for the 136 fast type (solid) and 150 slow type (dashed) solar wind discontinuities during 1998-2004. Thin solid line is a superposed epoch average for 1000 random time periods. (a) IMF magnitude, (b) solar wind density, (c) solar wind velocity, and (d) solar wind dynamic pressure.

\section{Upstream driver characterization}

Palmroth et al. (2007) studied sudden solar wind dynamic pressure pulses during the period 1998-2002. In Fig. 1 we present an overview of the data set with an expansion to the period 1998-2004. Stepwise sudden pressure pulses were identified the solar wind data by requiring that the pressure step was larger than $1 \mathrm{nPa}$ compared to a 30-min average prior to the pulse, and that the pressure fluctuations during the $30 \mathrm{~min}$ before and after the pulse were small. The second criterion was tested by requiring that the periods correlate with a Heaviside step function with a correlation coef- ficient of 0.95 or higher. The pressure pulses fall naturally into two categories: those for which the IMF magnitude simultaneously increases, and those for which IMF decreases. Following interplanetary shock theory, we named these event categories fast type discontinuities (FTD), and slow type discontinuities (STD), respectively. Figure 1 presents the superposed epoch averages for 136 FTD events (solid thick), for which the IMF magnitude (Fig. 1a) increases simultaneously with the pressure pulse (Fig. 1b-d). On the other hand, 150 STD events (dashed) with an IMF magnitude (Fig. 1a) decrease accompanying the impulse (Fig. 1b-d) were found. 

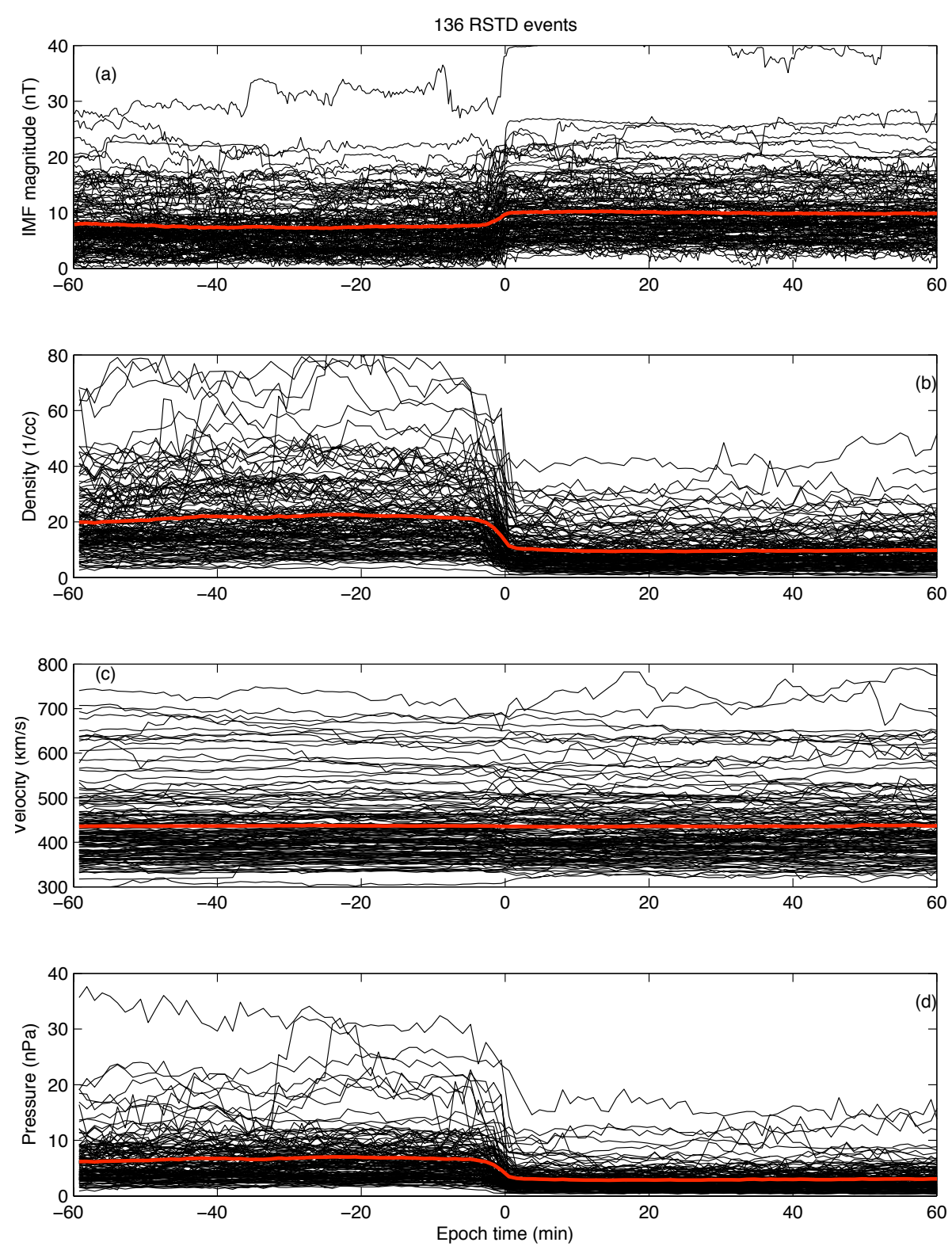

Fig. 2. Individual events (black) and the superposed epoch average (red) for the 136 sudden negative pressure pulses with a simultaneous increase in the IMF magnitude. (a) IMF magnitude, (b) solar wind density, (c) solar wind velocity, and (d) solar wind dynamics pressure.

We also identified a 1000 random time periods that serve as a data base for comparison between the selected pressure step events. Thin lines in Fig. 1 are the superposed epoch average from the random periods. Figure 1a indicates that the superposed epoch averages of the random periods represent the averages of the selected events: IMF increases from its typical values during the FTD events, while for the STD events the IMF returns to nominal after the pressure step. The solar wind density (Fig. 1b) for both the FTD and STD increase from the nominal values. The solar wind speed (Fig. 1c) during the FTD events is nominal, while the STD events occur during somewhat slower solar wind speeds. For both the FTD and STD events the dynamic pressure (Fig. 1d) increases from average values. As the random events give a representative averages of the data set, we conclude that the amount of events in the selected data set is sufficient. This indicates that possible errors (e.g., location of the IMAGE chain, the location of the upstream monitor and the time delay from ACE, the orientation of the front) are smoothed out in the statistics.

Figure 2 presents the solar wind conditions for sudden decreases of the dynamic pressure searched from the ACE 

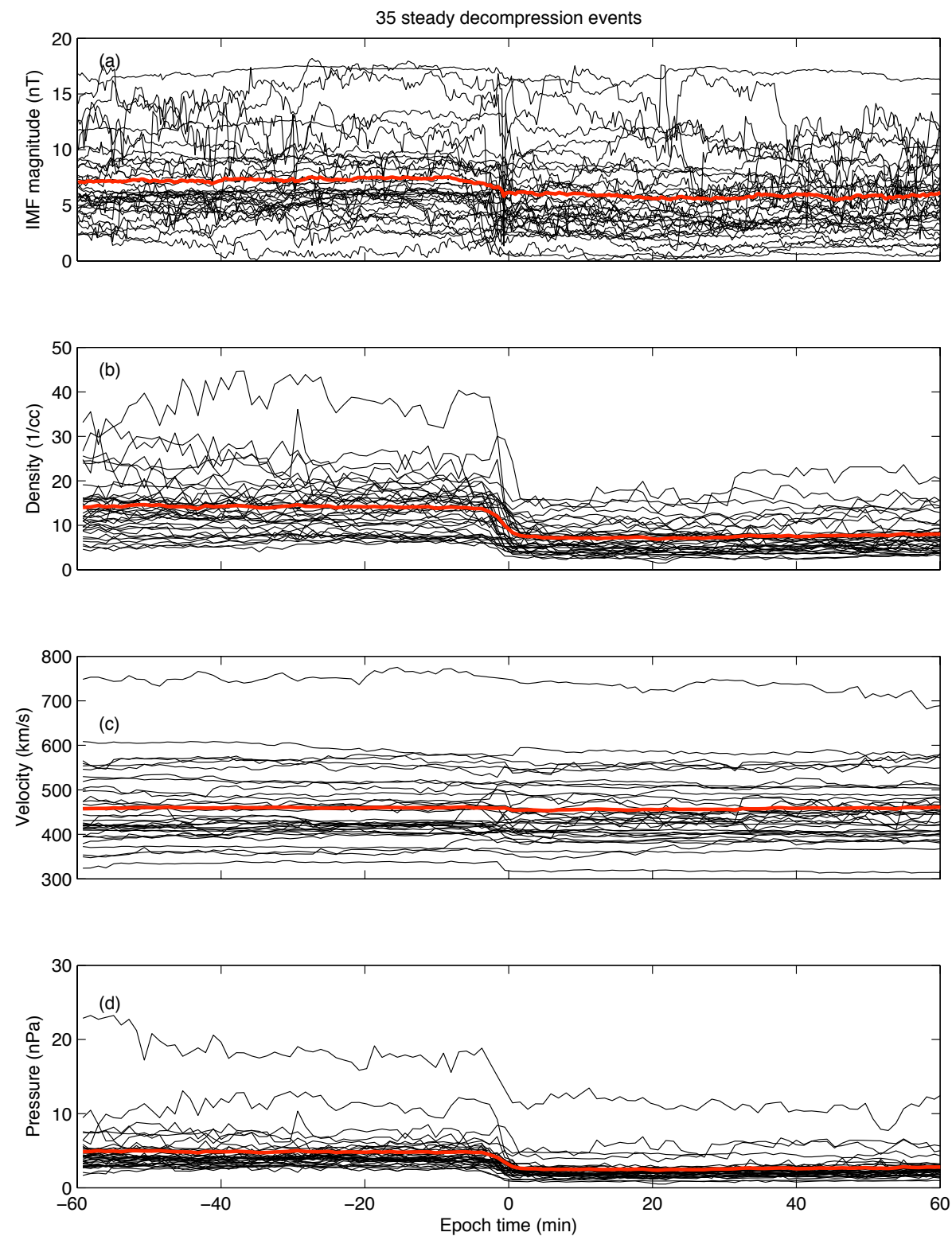

Fig. 3. Individual events (black) and the superposed epoch average (red) for the 35 steady decompression events with a simultaneous decrease or relatively steady IMF magnitude. (a) IMF magnitude, (b) solar wind density, (c) solar wind velocity, and (d) solar wind dynamics pressure.

recordings. The search procedure was similar as for the sudden increases of dynamic pressure in Fig. 1. A total of 171 negative pressure pulses were found during the period 1998-2004. Again the negative pressure pulse events were categorized according to the simultaneous IMF change, and in Fig. 2 we present cases for which the IMF magnitude (Fig. 2a) increases at the time of the negative pressure pulse (Fig. 2d). Individual events are shown in black while the red curve gives the superposed epoch average. The events are caused by the solar wind density (Fig. 2b) decreases, as the solar wind speed (Fig. 2c) is steady during the events. These events are termed reverse slow type discontinuities (RSTD) as their behavior resembles reverse slow shocks in the solar wind (Burlaga and Chao et al., 1971). A total of 136 RSTD events were found from the ACE recordings during the period $1998-2004$, indicating that $80 \%$ of all negative pressure pulses were of this type.

Figure 3 presents all other negative pressure pulse events (35 in total), of which some are accompanied with a small simultaneous decrease in the IMF magnitude (Fig. 3a), while others occur during relatively steady IMF conditions. For these events the solar wind density (Fig. 3b) decreases and 
Table 1. IE index response to pressure changes during different event types.

\begin{tabular}{lccccc}
\hline Event type & \# events & $\begin{array}{c}\overline{I E} \text { before } \\
(\mathrm{nT})\end{array}$ & $\begin{array}{c}I E_{\max } \text { after } \\
(\mathrm{nT})\end{array}$ & $\begin{array}{c}\Delta \\
(\mathrm{nT})\end{array}$ & $\begin{array}{c}\text { Change } \\
(\%)\end{array}$ \\
\hline FTD & 136 & 147 & 278 & +131 & 89 \\
FTD,$B_{z}>0$ & 71 & 108 & 194 & +86 & 80 \\
FTD,$B_{z}<0$ & 65 & 189 & 396 & +207 & 110 \\
STD & 150 & 172 & 206 & +34 & 20 \\
STD,$B_{z}>0$ & 76 & 91 & 118 & +27 & 30 \\
STD,$B_{z}<0$ & 74 & 255 & 307 & +52 & 20 \\
RSTD & 136 & 192 & $151^{*}$ & -41 & -21 \\
RSTD,$B_{z}>0$ & 72 & 138 & $97^{*}$ & -41 & $-30^{\mathrm{a}}$ \\
RSTD, $B_{z}<0$ & 64 & 253 & $211^{*}$ & -42 & -17 \\
\hline
\end{tabular}

*I $E_{\min }$

a No clear response in superposed epoch curves; the values are untrustworthy.

the solar wind velocity is unchanged (Fig. 3c), yielding a total decrease in the solar wind dynamic pressure (Fig. 3d). Lacking terminology originating from the shock theory, we term these events simply as steady decompression events.

\section{Ionospheric response}

In this section we study the response in the ionosphere during the events outlined in the previous section. The left panels in Fig. 4 presents the superposed epoch averages for the FTD (solid) and STD (dashed) categories, while the right panels are those for the RSTD (solid) and steady decompression (dashed) categories. Plotted are the solar wind $\epsilon$ (Fig. 4a and e), the IMF magnitude (Fig. $4 \mathrm{~b}$ and f), dynamic pressure (Fig. $4 \mathrm{c}$ and g), and the $I E$ index (Fig. $4 \mathrm{~d}$ and h). The figure presents our main result: the $I E$ index follows the evolution of the solar wind dynamic pressure regardless of the direction of change in the IMF or the $\epsilon$. The result is clear for all event categories except for the steady decompressions, where the effect is weak (reflecting possibly also the weak driver). The largest effect is seen in the FTD category, where the both the IMF and the dynamic pressure increase. Interestingly, the drivers for the RSTD and steady decompressions are opposite to each other: for the STD the IMF decreases and the dynamic pressure increases, but for the RSTD the opposite is true. Nevertheless the $I E$ follows the dynamic pressure and not the IMF or $\epsilon$.

Next we study the preconditioning hypothesis also touched by Liou et al. (2004). We separate the events in each category according to the average IMF direction $30 \mathrm{~min}$ prior the event to evaluate whether the ionospheric response is larger for those events for which energy has been accumulated in the tail during the prior southward IMF. Table 1 gives the average value of $I E$ superposed epoch average before the pressure change categorized according to the IMF north-south component, the maximum value to which $I E$ superposed epoch average is changed after the pressure step, and the change magnitude and percentage. The maximum of the $I E$ superposed epoch average is the maximum of the average of all individual events during a particular time period after the pressure step. Figure 5a-d presents the superposed epoch average for the $I E$ index for the FTD, STD, RSTD and steady decompressions, respectively. Solid (dashed) lines give the superposed average of $I E$ index for those events for which the IMF was on average southward (northward) for $30 \mathrm{~min}$ prior the pulse. Thin solid and thin dashed lines are the random events grouped similarly according to the IMF direction.

The $I E$ index increases for the FTD and STD events during both those events for which the IMF has been southward and for those for which the opposite is true (Fig. 5a-b). The response is augmented for the FTD events in both cases, as is also seen in Table 1. The southward IMF prior to the impulse makes the ionospheric effect larger for FTD events as the $I E$ increases $110 \%$ (80\%) for southward (northward) IMF (Table 1). Curiously, the opposite is true for the STD events, for which the $I E$ increases $30 \%$ for northward IMF and only $20 \%$ for southward IMF. The ionospheric response during the RSTD events decreases about $17 \%$ for southward IMF cases (Fig. 5c and Table 1). However, a clear change in the response during the RSTD events cannot be distinguished during northward IMF, and hence the 30\% decrease quoted in Table 1 may be unreliable. For the steady decompression events, there is a small ionospheric response in both IMF southward and northward cases (Fig. 5d). This reaction is smaller than the statistical fluctuations within the same curve, but larger than the fluctuations in the random events. However, as the reaction is so small, we have not specified the change associated to steady decompression events in Table 1. Comparing to the random events, it seems that the results are statistically significant.

Consequent to the preconditioning hypothesis, it has been suggested that the magnitude of the pressure step may be important in determining the amount of energy dissipated in the ionosphere (Burch, 1972). In Fig. 6a-d we study the effect caused by the magnitude of the pressure step for the FTD, STD, RSTD, and steady decompression categories, respectively. Solid (dashed) lines give the superposed averages of the $I E$ index for cases where the pressure step has been larger (smaller) than a threshold given in each panel. Random event cannot be used in this investigation as they do not include a pressure step. The pressure step size has been chosen such that the number of events in each category is roughly equal. The number of events falling into each category is given in parentheses in each of the legends. For all event categories except the steady decompressions, the larger pressure step creates a larger response in the ionosphere. The solid lines are also on higher level during the events, which possibly indicates that large pressure steps are likely to occur during disturbed times when the ionospheric activity is increased. 

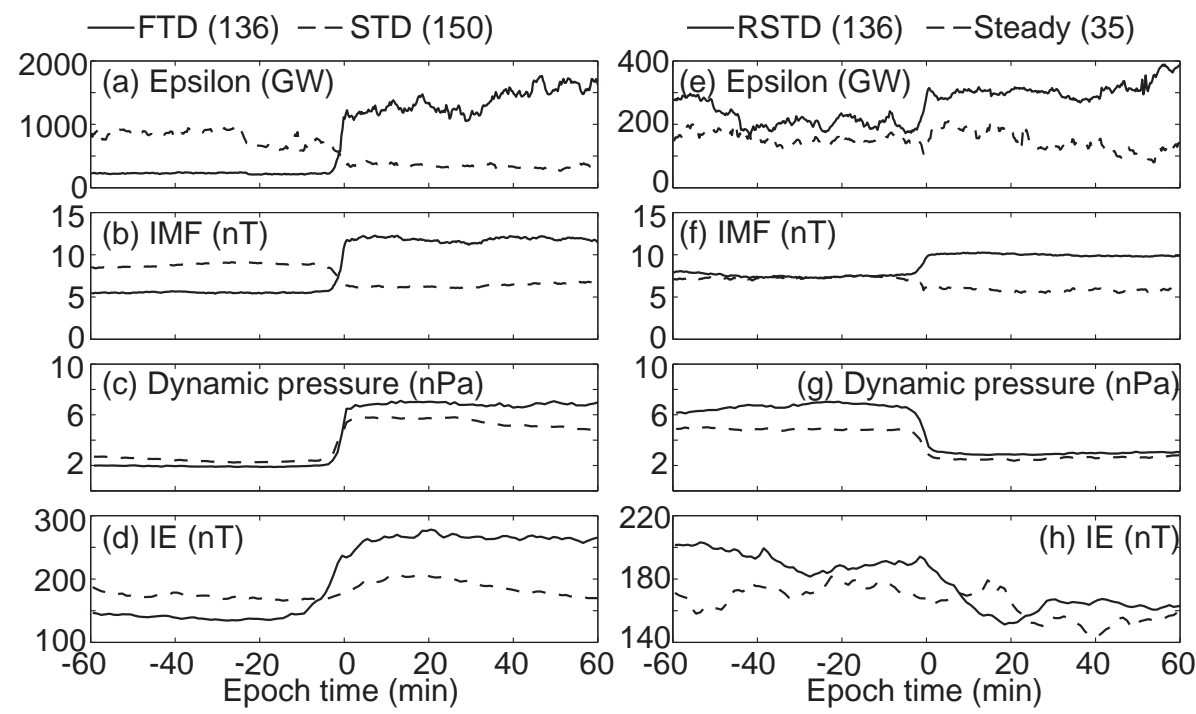

Fig. 4. Left panels: Ionospheric response to 136 fast type (solid) and 150 slow type (dashed) discontinuities during 1998-2004. Superposed epoch averages for (a) Solar wind epsilon, (b) IMF magnitude, (c) solar wind dynamic pressure, and (d) IE index derived from IMAGE magnetometer chain. Right panels: Ionospheric response to 136 sudden negative pressure pulses with a simultaneous increase in the IMF (solid) and 35 steady decompression events (dashed) during 1998-2004. Superposed epoch averages for (e) Solar wind epsilon, (f) IMF magnitude, (g) solar wind dynamic pressure, and (h) IE index derived from IMAGE magnetometer chain.
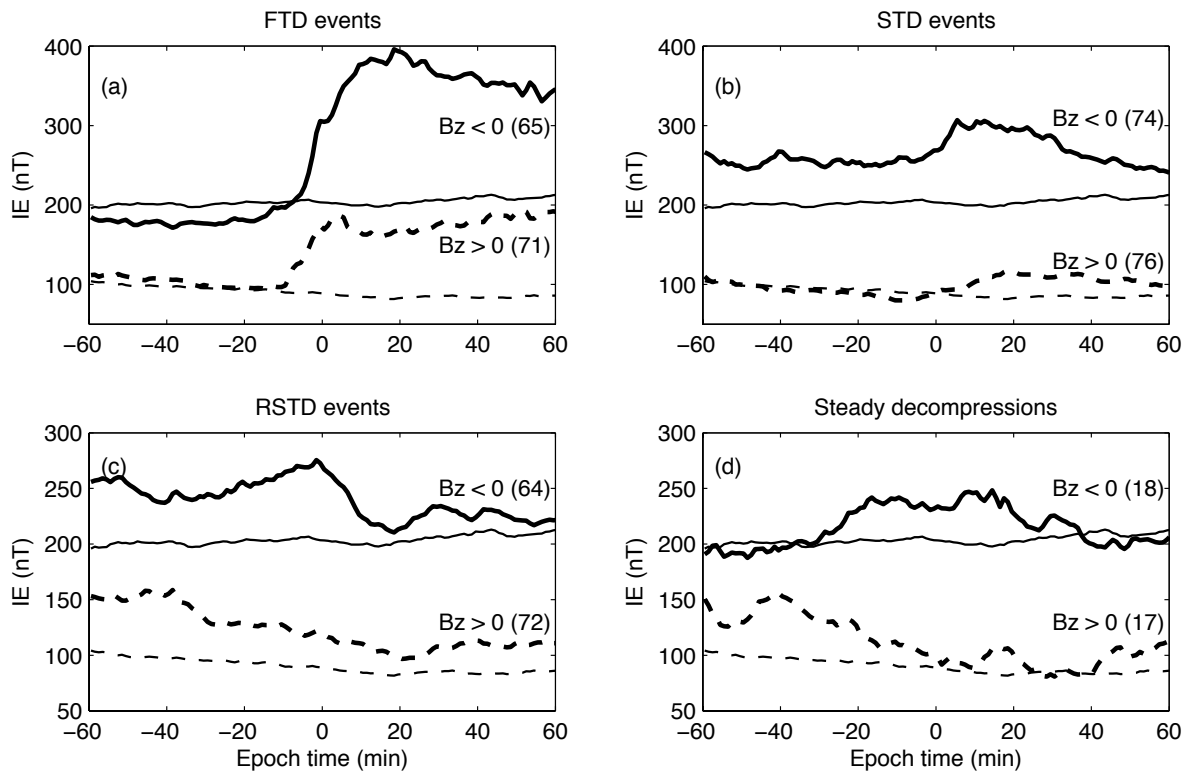

Fig. 5. Ionospheric response according to the average IMF direction $30 \mathrm{~min}$ prior to the event. Solid (dashed) lines show events for which the IMF has been southward (northward) for $30 \mathrm{~min}$ prior the epoch zero. Thin solid and thin dashed lines are the random events grouped similarly according to the IMF direction. Superposed epoch averages for the IE index for (a) FTD (b) STD, (c) RSTD and (d) steady decompressions are shown. Numbers in parentheses near each line show the amount of events in each category.

Figure 7 investigates whether the ionospheric dissipation depends on the dynamic pressure level, such that for higher pressures the ionospheric response would be different from lower pressure. Again, panels a-d are the $I E$ averages for the FTD, STD, RSTD, and steady decompression categories, respectively. Solid (dashed) lines give the superposed averages 

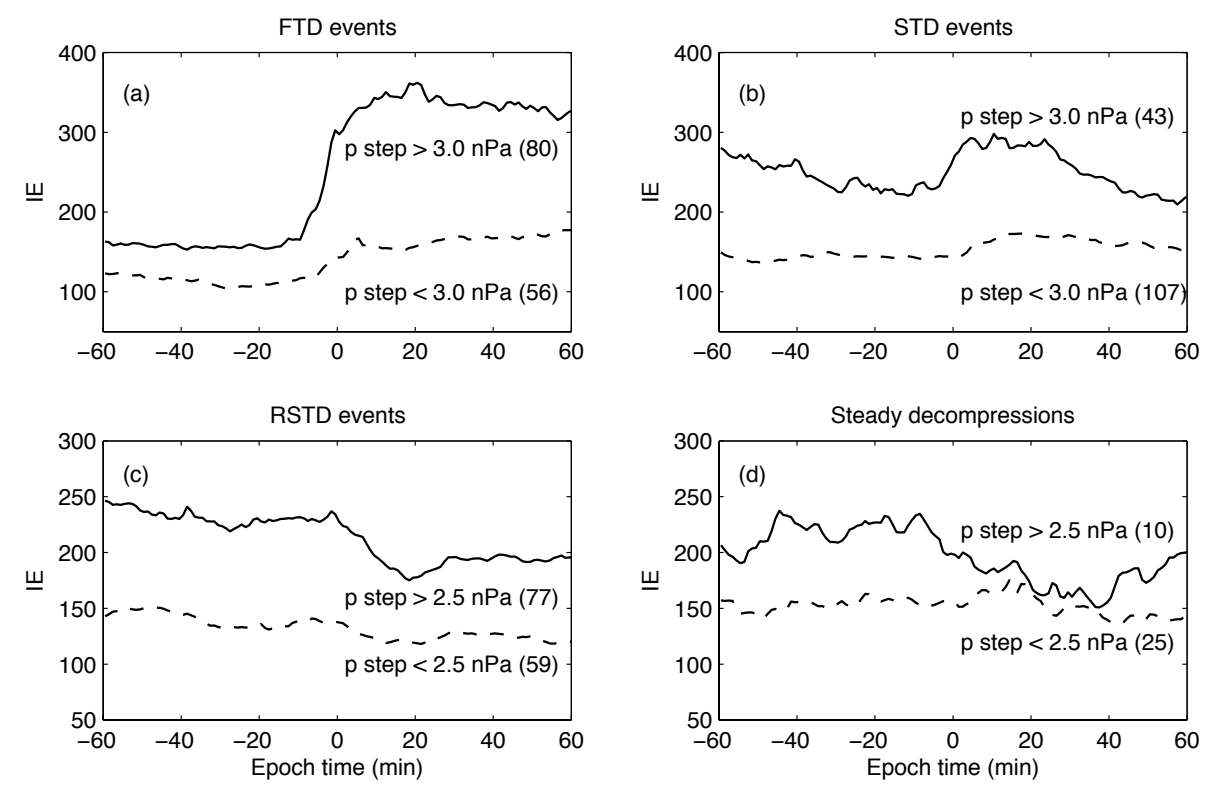

Fig. 6. Ionospheric response according to the dynamic pressure step size. Solid (dashed) lines show events for which the dynamic pressure step has been larger (smaller) than a threshold value given in each panel. Superposed epoch averages for the IE index for (a) FTD (b) STD, (c) RSTD and (d) steady decompressions are shown. Numbers in parentheses near each line show the amount of events in each category.

of the $I E$ index for cases where the pressure level has been larger (smaller) than a threshold given in each panel. Thin solid and thin dashed lines are the random events grouped similarly according to the pressure level. Comparison to the random events show that the results are statistically significant in all other categories except the steady decompressions. The pressure level prior the pressure step has a role only in the FTD category, and for all other event categories the $I E$ step size is roughly the same for both high and low previous pressures.

Finally, in Fig. 8 we investigate the effect of the IMAGE chain location. $I E$ is a local index, and the superposed epoch averages presented so far do not distinguish between the dayside and nightside effects. Again, panels a-d are the $I E$ averages for the FTD, STD, RSTD, and steady decompression categories, respectively. Solid (dashed) lines give the superposed averages of the $I E$ index for dayside (nightside) cases. Thin solid and thin dashed lines are the random events grouped similarly. The MLT range for the dayside events is 09:00-15:00 MLT and the nightside range is 21:00-03:00 LT. The $I E$ step during all event categories is roughly the same in both dayside and nightside, indicating that the ionospheric response is about the same over the ionosphere, suggesting that the results shown in Figs. 5-7 do not likely contain local time effects. The nightside superposed epoch averages are on a higher level than on the dayside, reflecting the fact that the westward electrojet is usually stronger than the eastward electrojet. The magnitude of the ionospheric response in the FTD and STD categories is well above the random cases increasing the statistical significance of the results.
The RSTD category shows a larger response in $I E$ than shown by the random events both in the dayside and nightside, giving credibility to the results. As a response to the steady decompression events in Fig. 8d, there is a distinct decrease of the $I E$ index on average at the time of the pressure step in the nightside (dashed line), although the magnitude of the effect is comparable to statistical fluctuations within the curve (at about $40 \mathrm{~min}$ before the pressure step). In addition, the curve is almost similar to the random events, decreasing the statistical significance of the result.

\section{Discussion and conclusions}

In this paper we have carried out the first statistical study of the ionospheric response to rapid increases and decreases in the solar wind dynamic pressure as manifested by the $I E$ index. As a distinction to Palmroth et al. (2007) we establish a new data set to investigate sudden decreases of the dynamic pressure as well as extend the previously used data set. We find here (as in Palmroth et al., 2007) that the pressure impulses searched from the solar wind recordings with our automatic routine comparing to a Heaviside function are often associated with simultaneous rapid changes in the IMF. This might reflect the nature of the solar wind where pressure changes are often associated with a discontinuity or a shock having a simultaneous change in the magnetic field. On the other hand, this might also be caused by our search routine that may favor events with large simultaneous fluctuations associated with solar wind discontinuities and shocks. 

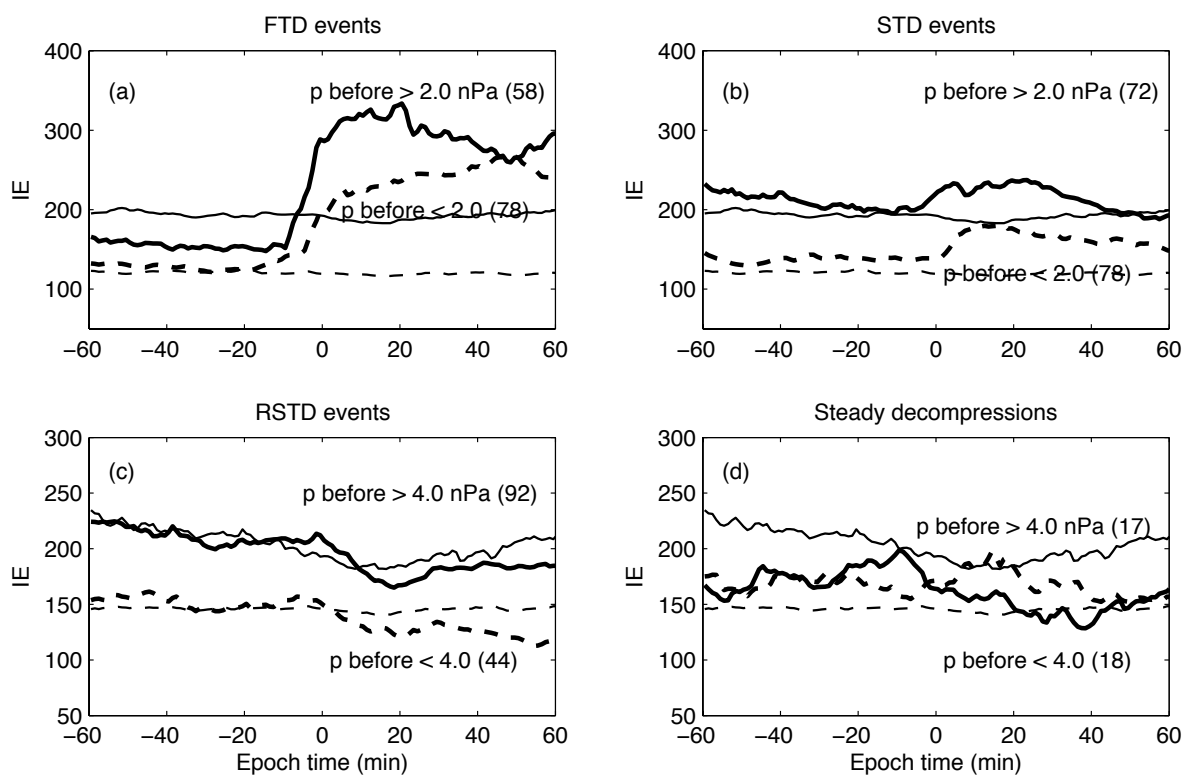

Fig. 7. Ionospheric response according to the dynamic pressure level before the pressure step. Solid (dashed) lines show events for which the dynamic pressure level has been larger (smaller) than a threshold value given in each panel. Thin solid and thin dashed lines are the random events grouped similarly according to the pressure level. Superposed epoch averages for the IE index for (a) FTD, (b) STD, (c) RSTD and (d) steady decompressions are shown. Numbers in parentheses near each line show the amount of events in each category.
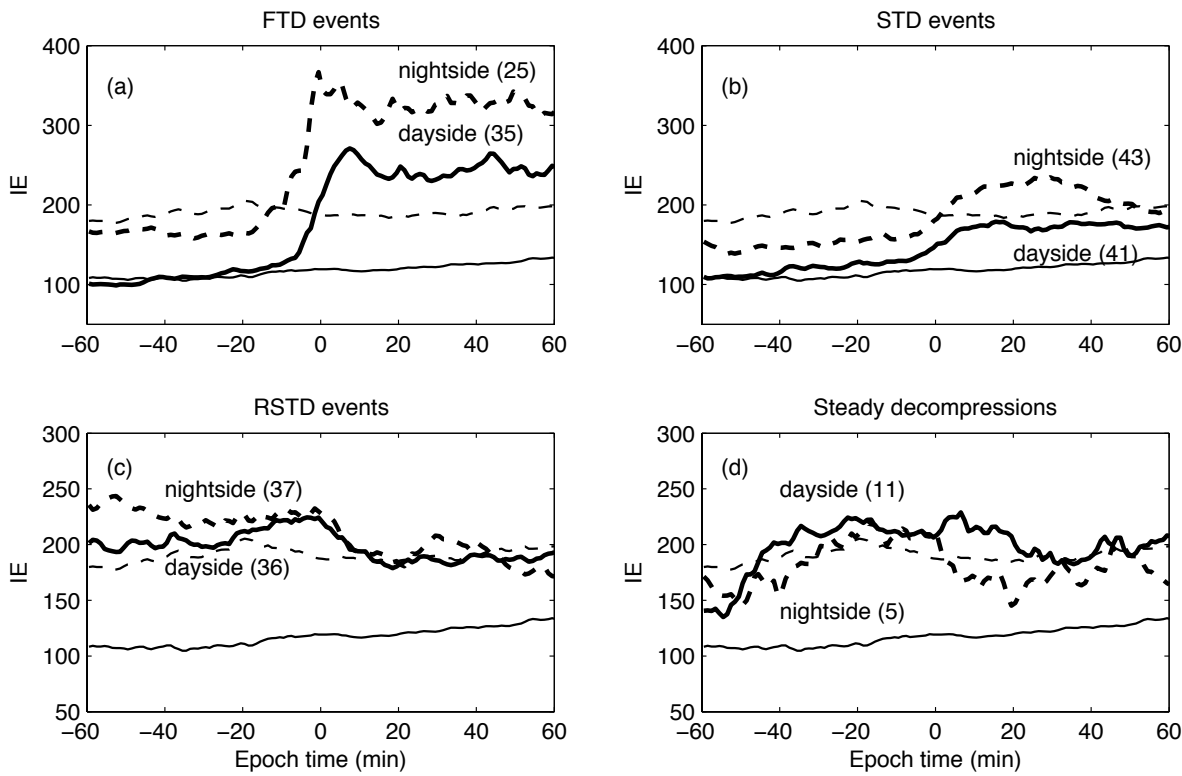

Fig. 8. Ionospheric response according to where the IMAGE chain was located at the time of the dynamic pressure step. Solid (dashed) lines show dayside (nightside) events. Events occurring within six hours around noon and midnight are allowed into each category. Thin solid and thin dashed lines are the random events grouped similarly according to MLT. Superposed epoch averages for the IE index for (a) FTD, (b) STD, (c) RSTD and (d) steady decompressions are shown. Numbers in parentheses near each line show the amount of events in each category. 
The drivers for roughly half of the pressure impulses resemble fast interplanetary shocks (FTD events) while the drivers for the other half resemble slow shocks (STD events). The drivers for the majority of the negative pressure pulses resemble reverse slow shocks for which the IMF simultaneously increases (RSTD category). We also find negative pressure pulse events for which the IMF stays relatively steady or is simultaneously decreasing. While some of the FTD and STD events fulfill the Rankine-Hugoniot relations and other shock tests (Palmroth et al., 2007), here we have not checked whether the events really are interplanetary shocks. From the four types of events, only the FTD category includes a simultaneous change in the solar wind density and velocity, while in the other categories the velocity stays constant and the density changes creating the dynamic pressure step. Especially interesting is to compare the response in the STD and RSTD categories, as for those events the solar wind drivers show a constant velocity while the density and the IMF changes are mirror images of each other: for STD events the density increases and IMF magnitude decreases, while for RSTD events the density decreases and IMF magnitude increases.

The main result of the paper is that in the statistically significant event categories the $I E$ increases as a response to increasing solar wind dynamic pressure, and decreases when the dynamic pressure decreases. The enhancement of the auroral electrojets as a response to positive pressure steps has been noted in several statistical and case studies (Shue and Kamide, 2001; Palmroth et al., 2004, 2007; Liou et al., 2004). The decrease of the $I E$ index as a response to the negative pressure step observed here is a new result in accordance with Liou et al. (2006), who found a decrease in the global auroral luminosity in a study containing 13 events. According to the results presented in this paper, the ionospheric response to a change in the solar wind dynamic pressure is an interplay between the dynamic pressure and the IMF: If the IMF and the dynamic pressure both increase (FTD events), the change in the $I E$ index is larger than for events where the dynamic pressure increases but the IMF decreases (STD events). If the dynamic pressure decreases and the IMF increases at the same time (RSTD events) the $I E$ index decreases, but the reaction is as weak as the response to the STD events. Consistent with the findings in Liou et al. (2004), it appears that the change in the dynamic pressure is more important than the simultaneous change in the IMF magnitude or in the $\epsilon$ parameter reflecting the energy input when it comes to the magnitude of the ionospheric response.

In comparing the STD and RSTD events, we find that the ionospheric response in these two data sets is of the same magnitude, but in the opposite direction: for STD events the $I E$ increases with the increasing pressure, while for the RSTD events the IE decreases with the decreasing pressure. Therefore our results indicate that the ionosphere is statistically "reversible" in response to the dynamic pressure changes. The symmetry in the responses between STD and
RSTD events is slightly surprising, as the mechanisms controlling the energy input in these events are different. For STD events, magnetopause reconnection (whether occurring in the lobes or at low latitudes) weakens as the IMF magnitude decreases, while the increase in pressure enhances the Chapman-Ferraro currents. For the RSTD events, reconnection enhances when the IMF increases while the ChapmanFerrraro currents weaken as the pressure decreases. This and the above indicate that the $I E$ changes follow the intensity of the Chapman-Ferraro current changes rather than responding to the changes in the magnetopause reconnection rate. Our results are consistent with Liou et al. (2004) who conclude that the changes in the auroral electrojets during interplanetary shocks are directly driven. We propose that the mechanism for the direct driving is through a connection between the Chapman-Ferraro currents and the Region 1 currents.

We have also investigated the ionospheric response after sudden negative pressure pulses that occur during relatively steady IMF. We find no particular response in the ionosphere in terms of the $I E$ index, whether grouped by the IMF direction or the pressure level preceding the pressure step, or the magnitude of the pressure step. A very weak effect is observed on events observed in the nightside. However, the data set is overall quite small containing only 35 events, and grouping makes the subsets even smaller, decreasing the statistical significance of the results. Furthermore, the drivers of the events are weak, as manifested by the superposed epoch averages in Fig. 3. We conclude that more events should be searched to this category in order to investigate the ionospheric effect statistically.

The identified data set also allows for the investigation of the preconditioning hypothesis. Grouping the events in the four categories according to the average IMF direction prior the pressure step shows that the ionospheric response is larger in the FTD and RSTD category, while there is no difference in the $I E$ step size in the STD and steady decompression category. Based on a smaller statistical data set, Liou et al. (2004) concluded that the preconditioning does not have a role in the ionospheric response. It is difficult to guess which type of events the Liou et al. (2004) data set included, but our findings indicate that possibly the structure of the driver determines the ability of the magnetosphere to extract energy form the solar wind. The same conclusion was drawn by Palmroth et al. (2007) who found that the efficiency of the ionospheric response is different between different drivers; more specifically that a weaker driver (STD events) is more geoefficient than a stronger driver. If this is true, it appears that the STD events are efficient in extracting the energy from the solar wind and hence in this category it makes no difference whether the magnetosphere is preconditioned or not. On the contrary, the other types of drivers are perhaps less efficient to extract the solar wind energy, and hence preconditioning magnifies the ionospheric response.

It has been speculated that the magnitude of the pressure step size determines the magnitude of the ionospheric 

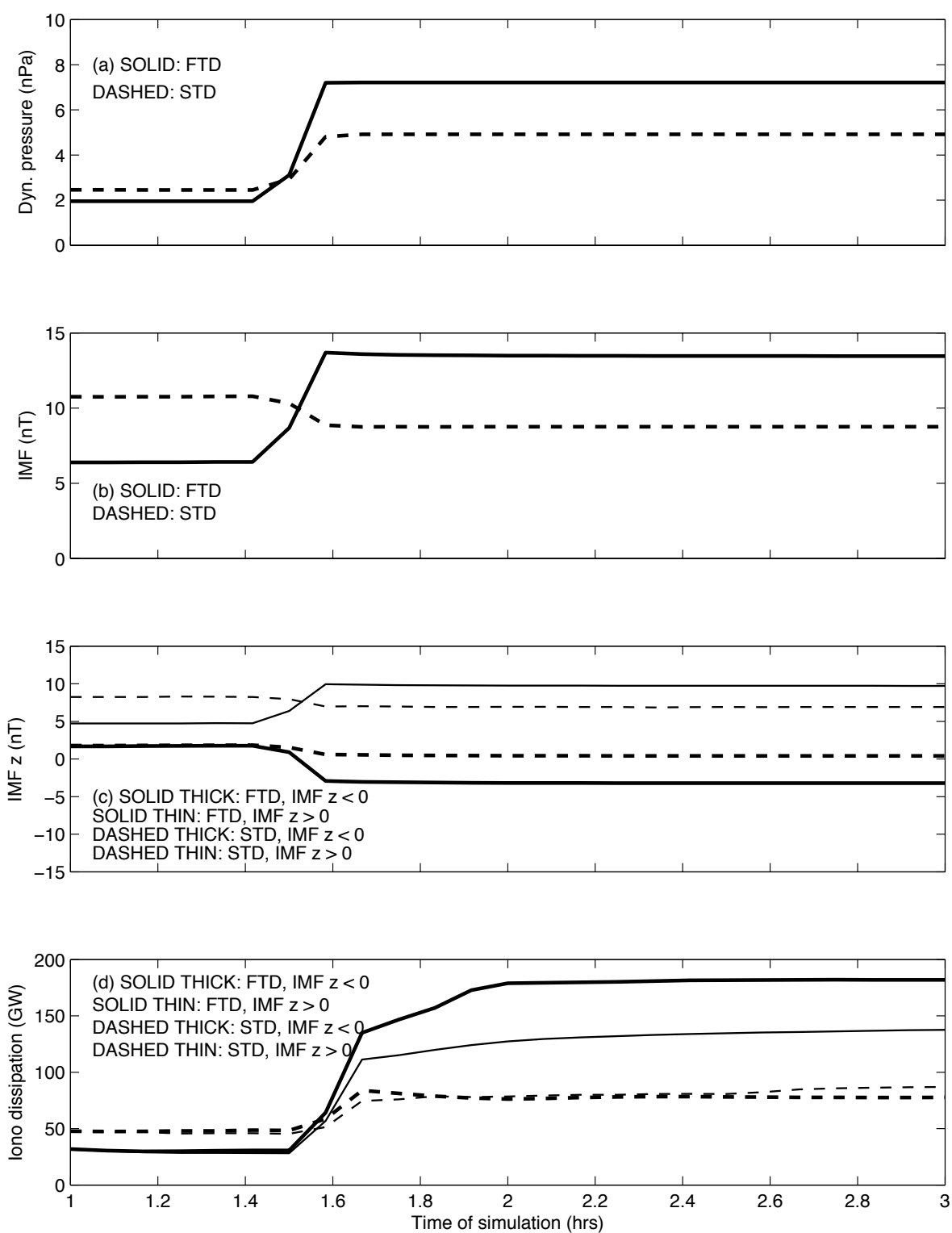

Fig. 9. Four simulation runs investigating the ionospheric response to FTD and STD events: (a) The solar wind dynamic pressure, (b) IMF, (c) IMF $z$ component, and (d) global ionospheric energy deposition. Solid thick (thin) lines indicate the FTD runs with IMF southward (northward) turning at the time of the impulse. Dashed thick (thin) lines indicate the STD runs with IMF southward (northward) turning at the time of the impulse. The dynamic pressure and the IMF magnitude are the same for the FTD and STD runs and hence the thin solid and dashed lines are not visible in panels (a) and (b).

response (Burch, 1972). Our findings support this idea: a larger pressure step in the FTD, STD and RSTD category yields a larger $I E$ step in the ionosphere: for the FTD and STD the $I E$ increases more, while for the RSTD it decreases more than for smaller pressure steps. This signifies our main result and suggests that the electrojet response is not only correlated with the solar wind dynamic pressure, but it is also proportional to the magnitude of the change.
Figure 9 investigates the ionospheric energy dissipation during FTD and STD events in a global magnetohydrodynamic (MHD) simulation GUMICS-4 (Janhunen, 1996). A total of four simulations were run: Two for an FTD event with IMF remaining positive and turning negative at the time of the impulse, and similarly two events for the STD events. The superposed epoch averages from the solar wind drivers of the FTD and STD events in this paper were used as inputs to the code: Solid and dashed lines in Fig. 9a show the 
dynamic pressure for the FTD and STD events, respectively, in the solar wind in the four events; while Fig. 9b shows the IMF magnitude. Figure 9c gives the evolution of the northsouth component of the IMF during the four runs: Thick and thin solid lines are the FTD events, while the thick and thin dashed lines are those for the STD events. Figure 9d gives the total ionospheric power consumption including the Joule heating and the electron precipitation power in Northern and Southern Hemispheres in the simulation with similar line types as in Fig. 9c. Clearly, the ionospheric dissipation increases as a response to both types of events, regardless of the simultaneous IMF change at the time of the impulse. We conclude that the GUMICS-4 MHD simulation results are in good agreement with the statistical analysis presented in this paper.

While the $I E$ index does not distinguish the directions of the ionospheric horizontal currents or convection, it is wellsuited to characterize the overall change in the ionosphere particularly in light of global energetics. The increase and decrease of the $I E$ index in concert with the dynamic pressure, regardless of the orientation of the IMF, is strong evidence of an ongoing energy dissipation process, manifested by Joule heating and electron precipitation. Similarly, the global ionospheric dissipation increases as a response to a dynamic pressure impulse in a global MHD simulation, even though the IMF turned more strongly northward at the time of the impulse. Based on the results shown in this paper, we argue that the ionospheric energy consumption is strongly related to the solar wind dynamic pressure, in addition to the well-known effect caused by the IMF.

Acknowledgements. The research leading to these results has received funding from the European Research Council under the European Community's Seventh Framework Programme (FP7/20072013) / ERC Starting Grant agreement number 200141-QuESpace. The work of MP is supported by the Academy of Finland. We thank all institutes maintaining the IMAGE magnetometer network (http://space.fmi.fi/image/).

Topical Editor I. A. Daglis thanks R. Lopez and two other anonymous referees for their help in evaluating this paper.

\section{References}

Akasofu, S.-I.: Energy coupling between the solar wind and the magnetosphere, Space Sci. Rev., 28, 121-190, 1981.

Araki, T.: Global structure of geomagnetic sudden commencements, Planet. Space Sci., 24, 373-384, 1976.

Araki, T. and Nagano, H.: Geomagnetic Response to Sudden Expansions of the Magnetosphere, J. Geophys. Res., 93(A5), 39833988, 1988.

Baumjohann, W., Bauer, O. H., Haerendel, G., Junginger, H., and Amata, E.: Magnetospheric Plasma Drifts During a Sudden Impulse, J. Geophys. Res., 88(A11), 9287-9289, 1983.

Boudouridis, A., Zesta, E., Lyons, L. R., Anderson, P. C., and Lummerzheim, D.: Enhanced solar wind geoeffectiveness after a sudden increase in dynamic pressure during southward IMF orientation, J. Geophys. Res., 110, A05214, doi:10.1029/2004JA010704, 2005.

Burch, J. L.: Preconditions for the triggering of polar magnetic substorms by storm sudden commencements, J. Geophys. Res., 77, 5629-5632, 1972.

Burlaga, L. F. and Chao, J. K,: Reverse and forward slow shocks in the solar wind, J. Geophys. Res., 76, 7516-7521, 1971.

Dungey, J.: Interplanetary magnetic field and auroral zones, Phys. Rev. Lett. , 6, 47-49, 1961.

Hubert, B., Palmroth, M., Laitinen, T. V., Janhunen, P., Milan, S. E., Grocott, A., Cowley, S. W. H., Pulkkinen, T. I., and Gérard, J.-C.: Compression of the Earth's magnetotail by interplanetary shocks directly drives transient magnetic flux closure, Geophys. Res. Lett., 33, L10105, doi:10.1029/2006GL026008, 2006.

Janhunen, P.: GUMICS-3: A global ionosphere-magnetosphere coupling simulation with high ionospheric resolution, in: Proceedings of Environmental Modelling for Space-Based Applications, 18-20 September 1996, Eur. Space Agency Spec. Publ., ESA SP-392, 1996.

Liou, K., Newell, P. T., Meng, C.-I, Wu, C.-C., and Lepping, R. P.: On the relationship between shock-induced polar magnetic bays and solar wind parameters, J. Geophys. Res., 109, A06306, doi:10.1029/2004JA010400, 2004.

Liou, K., Newell, P. T., Sotirelis, T., and Meng, C.-I.: Global auroral response to negative pressure impulses, Geophys. Res. Lett., 33, L11103, doi:10.1029/2006GL025933, 2006.

Lopez, R. E., Wiltberger, M., Hernandez, S., and Lyon, J. G.: Solar wind density control of energy transfer to the magnetosphere, Geophys. Res. Lett., 31, L08804, doi:10.1029/2003GL018780, 2004.

Lu, G., Onsager, T. G., Le, G., and Russell, C. T.: Ion injections and magnetic field oscillations near the high-latitude magnetopause associated with solar wind dynamic pressure enhancement, J. Geophys. Res., 109, A06208, doi:10.1029/2003JA010297, 2004.

Lukianova, R.: Magnetospheric response to sudden changes in solar wind dynamic pressure inferred from polar cap index, J. Geophys. Res., 108(A12), 1428, doi:10.1029/2002JA009790, 2003.

McComas, D. J., Bame, S. J., Barker, P., Feldman, W. C., Phillips, J. L., Riley, P., and Griffee, J. W.: Solar Wind Electron Proton Alpha Monitor (SWEPAM) for the Advanced Composition Explorer, Space Sci. Rev., 86, 563-612, 1998.

Palmroth, M., Pulkkinen, T. I., Janhunen, P., and Wu, C.-C.: Stormtime energy transfer in global MHD simulation, J. Geophys. Res., 108(A1), 1048, doi:10.1029/2002JA009446, 2003.

Palmroth, M., Pulkkinen, T. I., Janhunen, P., McComas, D. J., Smith, C. W., and Koskinen, H. E. J.: Role of solar wind dynamic pressure in driving ionospheric Joule heating, J. Geophys. Res., 109, A11302, doi:10.1029/2004JA010529, 2004.

Palmroth, M., Partamies, N., Polvi, J., Pulkkinen, T. I., McComas, D. J., Barnes, R. J., Stauning, P., Smith, C. W., Singer, H. J., and Vainio, R.: Solar wind-magnetosphere coupling efficiency for solar wind pressure impulses, Geophys. Res. Lett., 34, L11101, doi:10.1029/2006GL029059, 2007.

Sato, N., Murata, Y., Yamagishi, H., Yukimatu, A. S., Kikuchi, M., Watanabe, M., Makita, K., Yang, H., Liu, R, and Rich, F. J.: Enhancement of optical aurora triggered by the solar wind negative pressure impulse (SI?), Geophys. Res. Lett., 28(1), 127-130, 2001.

Shue, J.-H. and Kamide, Y.: Effects of solar wind density on auroral 
electrojets, Geophys. Res. Lett., 28, 2181-2184, 2001.

Shue, J.-H., Kamide, Y., and Gjerloev, J. W.: Effects of solar wind density on auroral electrojets and brightness under influence of substorms, Ann. Geophys., 27, 113-119, 2009,

http://www.ann-geophys.net/27/113/2009/.

Smith, C. W., Acuna, M. H., Burlaga, L. F., L'Heureux, J., Ness, N. F., and Scheifele, J.: The ACE magnetic fields experiment, Space Sci. Rev., 86, 613-632, 1998.

Stauning, P. and Troshichev, O. A.: Polar cap convection and PC index during sudden changes in solar wind dynamic pressure, J. Geophys. Res., 113, A08227, doi:10.1029/2007JA012783, 2008.
Vanhamäki, H., Amm, O., and Viljanen, A.: One-dimensional upward continuation of the ground magnetic field disturbance using spherical elementary current systems, Earth Planets Space, 55, 613-625, 2003.

Zesta, E., Singer, H. J., Lummerzheim, D., Russell, C. T., Lyons, L. R., and Brittnacher M. J.: The effect of the January 10, 1997 pressure pulse on the magnetosphere- ionosphere current system, in: Magnetospheric current systems, edited by: Ohtani, S.-I., Fujii, R., Hesse, M., and Lysak, R. L., American Geophysical Union Monographs, Washington, D.C., 217-226, 2000. 